\title{
Voglio Essere Io a Dire Come mi Chiamo: Nome e Paradigma IDENTITARIO NELLA LETTERATURA ITALIANA DELLA MigRAZIONE
}

\author{
VERA HorN
}

ABSTRACT Nel confrontarsi con l'altro e con la società di accoglienza, il migrante è portato ad un processo di rinegoziazione e ridefinizione del concetto d'identità, spesso dissipata, rinnegata, diluita in questo confronto. In questo senso, il nome, che costituisce uno degli aspetti della più complessa questione della formazione delle identità a seguito dei processi migratori, diviene un mezzo di affermazione e di salvaguardia dell'identità personale e culturale stessa contro l'appiattimento che può caratterizzare il processo d'inserimento e di integrazione sociale e che talvolta è rappresentato proprio nel tentativo di ribattezzare il migrante. La letteratura della migrazione in Italia, che può ormai vantare una produzione consistente che dura da quasi un ventennio, si è talvolta soffermata su questo problema nel suo percorso evolutivo, per mezzo di racconti, romanzi, anche di recente pubblicazione, di cui a seguire daremo notizia

PAROLE CHIAVE letteratura italiana della migrazione; identità; alterità; nome. 
REST $\mathrm{HO}$ Ao defrontar-se com o outro e a sociedade que o acolhe, o migrante é levado a realizar um processo de renegociação e de redefinição do conceito de identidade, freqüentemente dissipada, renegada, diluída nesse confronto. Nesse sentido, o nome, que constitui um dos aspectos da questão mais complexa da formação de identidade em conseqüência de processos migratórios, torna-se um meio de afirmação e de salvaguarda da identidade pessoal e cultural contra o achatamento que pode caracterizar o processo de inserção e integração social que, às vezes, é representado justamente na tentativa de rebatizar o migrante. A literatura da migração na Itália, que já tem uma produção consistente que dura há mais de vinte anos, deteve-se sobre esse problema em seu percurso evolutivo por meio de contos, romances, também recentes, dos quais daremos notícia a seguir.

PAIAVRAS CHAVE literatura italiana da migração; identidade; alteridade; nome

ABSTRACT On coming across the other and the society that welcomes them, migrants are led to renegotiate and redefine their concept of identity, often torn, disowned, diluted in this showdown. In this sense, the name, that is one of the aspects of the complex issue of identity formation as a result of migration processes, becomes a means to reaffirm and safeguard personal and cultural identity against the loss that can occur during the process of social insertion and integration that sometimes attempts to rebaptize migrants. Migration literature in Italy, which already relies on a consistent production spanning over twenty years, dwelt on this problem in the course of its evolution as seen in recent tales and novels mentioned below.

IEYWORDS Italian migration literature; identity; alterity; name 
Right off the boat, or Boeing, I admit being tongue-tied.

For I am the language that is lost,

The name that is changed.

(...)

Mother tongue in mind, adopted tongue in cheek. You were Commonwealth; I am common loss.

(Raymond Filip, The Mighty Buck, the Immigrant Fuck, and Melting Pot Luck)

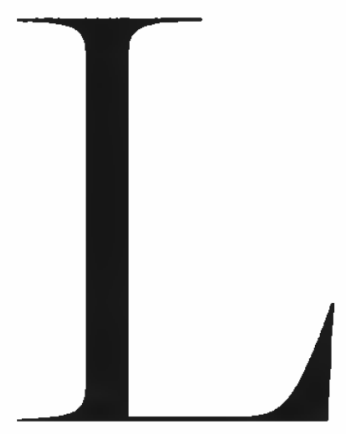

a frase iniziale del titolo riprende le parole dell'antropologa camerunense radicata in Italia Geneviève Makaping, che nel suo Traiettorie di sguardi afferma: "voglio dire io come mi chiamo [...] non devi dirmi tu il mio nome"1. Questa perentoria affermazione della propria identità assume un significato ben preciso in un contesto immigratorio: nel confronto con l'Altroo con la società di accoglienza, il migrante è portato ad un processo di rinegoziazione e ridefinizione del concetto di identità, spesso repressa, diluita, appiattita nei processi di inserimento e di integrazione sociale. Il nome, naturalmente, costituisce un aspetto soltanto della questione, ben più complessa, della formazione delle identità a seguito dei processi di emigrazione-immigrazione.

Il contatto del migrante con la società di accoglienza, nella fattispecie italiana, genera spesso fenomeni di stereotipazione e di stigmatizzazione, di cui sono esempi notil'attribuzione dell'aggettivo patrio "marocchini" a qualsiasi immigrato di origine africana, talvolta con l'aggiunta di "neri" per i soggetti dalla pelle più scura, e l'assegnazione vaga e generalizzata di "vu' cumprà" 2 come mestiere tipico e indispensabile del migrante, anche in questo caso spesso di origine africana. Fortemente collegata alla stereotipazione della figura del migrante è la questione fondamentale del nome. I nomi degli immigrati, ritenuti "difficili". vengono scambiati con nomi italiani, talvolta per assonanza, anche lontana, talvolta per arbitraria imposizione da parte di parlanti italiani: così Ahmed diventa Amedeo, Karim diventa Gianni, Abdul diventa Marco, Taufik diventa Vito. Ma la questione va al di là della semplice difficoltà di pronuncia.

Il racconto Io marocchino con due Kappa (1995) dell'allora esordiente Yousef Wakkas, premiato nel primo concorso Eks\&Tra, aveva colto subito la questione:

1. Geneviève Makaping, Traiettorie di sguardi. E se glialtri foste voi?, Soveria Mannelli, Rubbettino, 2001, pp. 53-54.

2. Con questa espressione, dalle sfumature spregiative, vengono chiamati in Italia i venditori ambulanti immigrati. Secondo P. Restiotto e A. Micheletti, la parola è apparsa per la prima volta nel 1925, in $O^{\prime}$ Tripolino di Raffaele Viviani, riferita ad un italiano trasferitosi in Libia come venditore ambulante. Solitamente viene interpretata come una contaminazione del francese vous con l'italiano comperare. Cfr. P. Restiotto e A. Micheletti, "Materiali di lavoro", in Saidou Moussa Ba e A. Micheletti, La promessa di Hamadi, Novara, De Agostini, 2006 [1991], p. 157. Una delle opere inaugurali della letteratura della migrazione, Io venditore di elefanti (Garzanti, 1990) di Pap Khouma, racconta per l'appunto la storia di un venditore ambulante senegalese alla ricerca delle piazze più convenienti per vendere la propria merce, simboleggiata dagli elefanti del titolo, dalle spiagge della riviera romagnola ai mezzanini del metrò milanese, e naturalmente non immune alle aggettivazioni generalizzate e alle problematiche identitarie. 
[...] come si chiama?" poi ha strizzatol'occhio maliziosamente a Paola. "Abdulfattah" io ho anticipato Paola nel rispondere. "Abdul Fattah! e questo sarebbe un nome?" ha esclamato la seconda, ma la prima ragazza ha trovato subito la soluzione: "Perché non lo chiamiamo Marco?" io non ho dimostrato nessuna obbiezione, ormai è diffusa l'abitudine di chiamarci con nomi familiari, Mustafa diventava Mino, e Hussein diventava Enzo. ${ }^{3}$

Il desiderio di rendere familiare il nome "esotico" è quindi la spiegazione per il cambio del nome dell'immigrato. Questa strategia ha, però, dei risvolti più articolati, in quanto il nome dell'immigrato si carica di presupposti identitari. In un contesto immigratorio il nome, che, come sottolinea Gisella Brinkler-Gabler, è ovviamente un "powerful sign of identity"4, può essere una remora all'integrazione, può funzionare da ostacolo nel reciproco riconoscimento: come il mare del poema Orizzonte di Fernando Pessoa lascia solo sperare oltre la fredda linea dell'orizzonte l'albero, la spiaggia, il fiore, l'uccello, la fonte, che si trovano attraversandolo ${ }^{5}$; e soltanto una volta svelati, con sensibili movimenti di speranza e di volontà, il mare e il mistero, ciò che era timorosamente percepito come estraneo diventa familiare e non arreca più timore. Tuttavia, come abbiamo detto, la questione è più complessa. Per "Marco". questo scambio del nome viene associato ad altri episodi che nel loro insieme fanno riferimento alla visione negativa dell'altro, alla formazione degli stereotipi e alla difficoltà di inserimento:

- Quando l'hai conosciuto?

- Due settimane fa.

Per la verità, era passato più di un mese da quando avevo visto Paola per la prima volta. Questa sua dichiarazione timida di dimostrare un distacco leggero da me quando ci incontravamo con gli amici o con i suoi parenti, è proseguita cosi durante tutta la nostra relazione fino all'ultimo giorno. Il desiderio si contraddiceva con la paura dei rimproveri che traboccavano negli occhi delle persone che ci scrutavano con sguardi diffidenti durante la nostre apparizioni in strada e nei locali pubblici. D'altronde, anche suo padre ha espresso sempre a voce alta la sua opinione, come ha fatto subito dopo il nostro rientro da un week-end fuori Milano. Eravamo sul marciapiede davanti al portone di casa, e rivolgendosi al panettiere disse: "Gente senza radici, altrimenti non avrebbero lasciato il loro paese."

Gli aspetti dell'identità, specificamente razziale, sono costruiti attraverso lo sguardo dell'autoctono:

Ho sentito per la prima volta la parola "Marocchino" e anche qualche parolaccia, e ho letto sui muri frasi di "accoglienza" verso di noi, e ho visto i bambini attaccarsi alle gambe della loro madre quando salivamo sui mezzi pubblici.?

3. Yousek Wakkas, "Io marocchino con due kappa”, in R Sangiorgi e A. Ramberti, Le voci dell'arcobaleno, Santarcangelo di Romagna, Fara, 1995, p. 143 4. Gisella Brinkler-Gabler, Encountering the other(s). Studies in Literature, History, and Culture, Albany, S'

5.Ó mar anterior a nós, teus medos

Tinham coral e praias e arvoredos. Desvendadas a noite e a cerraçāo,

As tormentas passadas e o mistério,// Abria em flor o Longe, e o Sul sidério

'Splendia sobre as naus da iniciação. Linha severa da longinqua costa -

Quando a nau se aproxima ergue-se a encosta//

6. Y. Wakkas, op. cit., pp. 143-144.

7. Ibidem, p. 137.

\section{Em árvores onde o Longe nada tinha;} Mais perto, abre-se a terra em sons e cores: E, no desembarcar, há aves, flores, Onde era só, de longe a abstracta linha// $O$ sonho é ver as formas invisiveis Da distância imprecisa, e, com sensiveis Movimentos da esp'rança e da vontade, Buscar na linha fria do horizonte//
A árvore, a praia, a flor, a ave, a fonte Os beijos merecidos da Verdade.

(Fernando Pessoa, "Horizonte", in Oeu profundo e os outros eus, Rio de Janeiro, Aguilar, 1972, p. 53) 
Lapresenza del migrante turbal'ordine sociale precostituito e costringe ad una nuova rielaborazione dell'assetto sociale e dell'ordine culturale: "nel riconoscimento dell'altro [...] riconosciamo di non essere più al centro del mondo"8

Anche nel romanzo Fiamme in paradiso (2000) di Smari Abdel Malek, il protagonista Karim si sente espropriare la propria identità quando gli viene proposto il nome Gianni al posto del suo. La storia di Karim è quella di tanti altri immigrati raccontati nelle pagine della letteratura migrante: attirato dal mito occidentale dell'abbondanza e della ricchezza, decide di emigrare in Italia alla ricerca di una situazione migliore, non vedendo prospettive nel suo paese, l'Algeria:

qui non ho più nessun interesse. Nessun lavoro, un'attività mia, neanche uno straccio di speranza di lavoro. ${ }^{9}$

Karim è quindi portato alla scelta quasi obbligata di emigrare. Tuttavia, in Italia, lui non troverà i presupposti che si aspettava per poter realizzare il suo progetto immigratorio, e vedrà rapidamente infrangersi le sue speranze; quindi, senza lavoro e senza casa, Karim intraprende la via della clandestinità e della vita ai margini della società. Ciononostante, lui non si scoraggia e desidera restare in Italia. La situazione di Karim sovverte la consueta equazione migrantenostalgia ( $g$ hurba o ghorba), che è solitamente associata alla letteratura della migrazione, di qualsiasi latitudine, come uno dei suoi principali temi:

In verità Karim non sentiva nessuna nostalgia per le persone né per le cose né per $\mathrm{i}$ luoghi. Se per nostalgia si intende il desiderio del ritorno, lui non voleva tornare.Gli venne in mente il buio che copriva il cielo a Costantina il giorno della sua partenza. Il paese del buio, cosi gli era sembrato allora il suo paese. No, non voleva tornare. ${ }^{10}$

Per il protagonista, però, questo desiderio non si traduce nella accettazione passiva della fagocitazione della sua identità dalla parte dell'autoctono. In effetti, la proposta di venire chiamato Gianni perché "è più semplice" viene percepita da Karim come una perdita d'identità:

"Perdo un altro pezzo" pensò Karim "il nome!"”l

La perdita del tratto identitario, percepita dal protagonista come negazione, come "vergogna e umiliazione", non si risolverà mai nella trama in modo positivo; in effetti, dopo l'esplosione di un'autobomba che provoca la morte di Karim, passante incauto al momento della terribile tragedia, i giornali annunciano: "Quattro persone e un marocchino morti in un attentato in via Palestro.

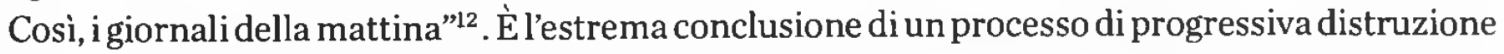
dell'identità dell'immigrato, che ha perso ormai il nome, la nazionalità (per gli italiani, "tutti

8. Iain Chambers, Paesaggi migratori. Cultura e identità nell'epoca postcoloniale, Genova, Costa e Nolan, 1996, pp. 28-29. 9. Smari Abdel Malek, Fiamme in paradiso, Milano, Il Saggiatore, 2000, p. 10.

10. Ibidem, p. 67.

11. Ibidem, p. 143.

12. Ibidem, p. 158. 
sono marocchini”'13 aveva osservato Karim) e da ultimo la sua personalità, la sua umanità. Anche Mohamed Bouchane, fin dal titolo del suo Chiamatemi All (1991), denuncia la questione. Bouchane registra in un diario tutte le tappe della sua vita da immigrato in Italia, precisamente a Milano, dalla clandestinità alla sanatoria. Nelle diverse tappe della sua vita da immigrato, lo scrittore non tralascia in nessun momento di riferire il suo profondo legame con la religione (musulmana) e come il fatto di trovarsi in moschea o nel Centro Islamico, tra persone che professano la stessa fede e parlano la stessa lingua, si traduca effettivamente nel senso di casa per chi una casa non ce l'ha:

Sono passato dalla vita serena che facevo in Marocco quando studiavo biologia all'università di Rabat a una vita da barbone. Nella mia città avevo visto spesso gente senza casa, costretta ad arrangiarsi per dormire e per mangiare. Ma non avrei mai immaginato che sarei diventato come loro. ${ }^{14}$

La convivenza con gli italiani, anche se pacifica, dimostra i pregiudizi e gli stereotipi che di solito si rivolgono alla cultura dell'altro:

come sempre, il discorso finisce sul Marocco. Quando dico che è un paese aquattordici chilometri dalla Spagna non mi credono. E non mi credono nemmeno quando dico che non ci sono cammelli fermi ai semafori. [...] Maurizio e Carlo [...] pensavano che in Marocco ci fossero i cammelli al posto delle macchine. ${ }^{15}$

Per Mohamed la problematica del nome ha due risvolti ben precisi. Il primo si riferisce alla sostituzione del suo nome con l'appellativo "bestia", che un collega di lavoro, "un muratore bresciano di sessantun'anni", gli rivolge, solitamente in presenza di altri, il che evidenzia il desiderio di umiliare, di disprezzare. Il secondo, invece, si riferisce alla solita problematica della pronuncia. Tale episodio è ricorrente, ma Mohamed rifiuta di venire chiamato con un nome italiano:

Prima che mi affibbino un nome italiano - Taufik una volta è stato soprannominato Vito! - passo al contrattacco. "Se proprio non riuscite a chiamarmi Mohamed" dico "allora chiamatemi Ali”. Scelgo Alì perchéè semplice e, come Mohamed, è un nome molto amato e molto diffuso in Marocco. ${ }^{16}$

Dopo la sanatoria, che come spiega l'io narrante, restituisce la dignità di cittadino all'immigrato Mohamed Bouchane, l'episodio si ripete:

il principale non riesce mai a chiamarmi Mohamed: si confonde, pasticcia, si ferma, lo storpia. ${ }^{17}$

13. Ibidem, p. 84.

14. Mohamed Bouchane, Chiamatemi All, Milano, Leonardo, 1991, p. 27.

15. Ibidem, p. 78.

16. Ibidem, p. 155

17. Ibidem, p. 183. 
Mohamed propone nuovamente di venire chiamato Ali, cosi "tutto è più semplice". Il nome tramutato viene situato nello stesso contesto del nome originale e riesce pertanto a conservare una parte della sua identità.

La nominazioneè infatti uno strumento primordiale e fondamentale di appropriazione: Todorov, riferendosi alla conquista dell'America, identifica in Colombo una continua sete di nominare. Colombo sa che le isole possiedono già un nome, ma i nomi degli altri non gli interessano. Lui desidera ribattezzare $\mathrm{i}$ luoghi in funzione del posto che occupano nel quadro della sua scoperta $\mathrm{e}$ dare loro nomi "giusti". In questo caso, sottolinea Todorov, il processo di nominare ha il significato di prendere possesso ${ }^{18}$ Tale concetto è condiviso da Iain Chambers, secondo il quale "nominare è possedere [...]. Di solito siamo disposti a riconoscere le differenze purché rimangano entro i confini del nostro linguaggio, del nostro sapere, del nostro controllo"19

Il caso del romanzo di Amara Lakhous, Scontro di civiltà per un ascensore a Piazza Vittorio (2006), è diverso dagli altri finora esaminati. Piazza Vittorio e il condominio che corrisponde all'ambientazione principale del romanzo fungono da piccolo campione dell'Italia odierna vista da uno sguardo doppio che guarda al multiculturalismo e alle sue conseguenze presenti e future nella fisionomia italiana, sia in ambito culturale (lingua), che politico-sociale (integrazione e questioni relative; diritto e cittadinanza); la consapevolezza culturale del romanzo di Lakhous è molto maggiore che nei più precoci esempi che abbiamo esaminato, come si vede dalla trattazione di questo tema. In questo romanzo il diverso atteggiamento di due immigrati, Ahmed e Abdallah, di fronte alle difficoltà materiali e psicologiche dell'immigrazione viene efficacemente riassunto nella reazione al nuovo, imposto battesimo. Il protagonista Ahmed ha il nome cambiato in un nome italiano, Amedeo, però al contrario degli altri immigrati, non si ribella al fatto, come invece il suo connazionale Abdallah Ben Kadour, anche lui immigrato. Abdallah racconta di come gli hanno proposto "una serie di nomi italiani come Alessandro, Francesco, Massimiliano, Guido, Mario, Luca, Pietro e altri ancora" ${ }^{20}$, tutti decisamente rifiutati. Secondo Abdallah, molti immigrati lo fanno "per far piacere agli italiani", d'altronde in corrispondenza con un verso del Corano secondo il quale "Gli ebrei e i cristiani non ti accetteranno finché non seguirai la loro religione"21 Il nome, per Abdallah, è profondamente radicato nel concetto d'identità:

Non cambierò pelle, né religione, né il mio paese, né il mio nome per nessuna ragione. ${ }^{22}$

Invece Ahmed, immigrato algerino, diventa Amedeo, percepito dagli italiani e dagli stessi immigrati come italiano a tutti gli effetti, come si avverte dalle parole di un suo conoscente bengalese, anch'egli immigrato:

Il signor Amedeo è un italiano diverso dagli altri: non è fascista, voglio dire non è un razzista che odia gli stranieri. ${ }^{23}$

18. Tzetan Todorov, La conquista dell'America. Il problema dell'altro, trad. Aldo Serafini, Torino, Einaudi, 1992, p. 33.

19. Chambers, op. cit., p. 35.

20. Amara Lakhous, Scontro di civiltà per un ascensore a Piazza Vittorio, Roma, e/o, 2006, p. 159.

21. Ibidem, p. 16l. Il testo della Sura II Al-Baqara (La Giovenca) del Corano recita: "Né i giudeí né i nazareni saranno mai soddisfatti di te, finché non seguirai la loro religione"

22. Ibidem, p. 161.

23. Ibidem, p. 61. 
Ma anche ipersonaggi italianidel romanzo lopercepiscono come un italiano e reagiscono con sorpresa quando lo scoprono immigrato, come la portinaia del palazzo dove abita, Benedetta Esposito:

Che dite? Il signor Amedeo è forestiero? Non ci credo che non è italiano! Non ho ancora perso la testa, sono in grado di distinguere tra gli italiani e gli stranieri. [...] Perché insistete? Vi ho detto che Amedeo è italiano verace. ${ }^{24}$

Perfino il commissario Bettarini, che indaga sull'assassinio avvenuto nell'ascensore del condominio dove risiede Amedeo e la maggior parte degli altri personaggi, aveva creduto che AhmedAmedeo "fosse un volontario italiano che aiutava gli immigrati"25

Ben consapevole del valore paradigmatico dell'episodio, Lakhous non tralascia di chiarire l'origine del cambio di nome, che non implica imposture né tradimenti, ma ironicamente chiama in causa il dialetto, il romanesco di Sandro Dandini, il proprietario del bar frequentato da Ahmed-Amedeo; quando Sandro chiede ad Ahmed il suo nome, capisce "Amed", che poi diventa "Amedé, con la consueta integrazione romanesca delle finali. Ahmed diventa Amedeo quasi senza volerlo ma senza opporvisi, con una naturalezza che copre però risvolti identitari ben precisi. Lui ha un passato doloroso (in Algeria ha perso la fidanzata Bágia, uccisa dai fondamentalisti) che non vuole vedere riemergere e in questo caso, conclude, dopo la lettura di Leone l'africano di Amin Maalouf, che "è meraviglioso potersi liberare dalle catene dell'identità che ci portano alla rovina" ${ }^{26}$. Ahmed desidera "farsi allattare dalla lupa" senza farsi mordere da essa. Quest'espressione era, tra l'altro, parte del titolo del romanzo pubblicato prima in Algeria, nel 2003 - l'edizione italiana è del 2006 - Come farti allattare dalla lupa senza che ti morda, che mostra bene come si proponga nel libro una strategia di assimilazione che prevede la sovrapposizione di una identità immigrata a quella originale e alle (terribili, in quel caso) esperienze precedenti, non tuttavia senza residui, evidenti anche nella struttura narrativa. Il nome italiano permette a Ahmed di inserirsi facilmente e ricostruirsi una vita italiana, grazie anche alla padronanza perfetta della lingua: "l'italiano è il mio latte quotidiano"27

Il paradigma inaugurato con l'indolore e incolpevole cambio di nome si realizza anche su altri piani. Ahmed, diventato Amedeo, oltre alla perfetta padronanza della lingua, può vantare un'esemplare conoscenza della cultura e del territorio:

Conosce la storia di Roma e le sue strade meglio di me, anzi meglio di Riccardo Nardi, fierissimo delle sue origini che risalgono agli antichi romani. Riccardo il tassista, che attraversa le strade di Roma su e giù ogni giorno da vent'anni. Una volta ho fatto a gara con Amedeo a chi conoscesse le strade e io come un presentatore di quiz televisivi ponevo loro una serie di domande, ad esempio: dove si trova via 
Sandro Veronese? Dove si trova via Valsolda? Come si arriva da piazza del Popolo a via Spartaco? Dove si trova piazza Trilussa? E piazzale della Radio? E il ministero degli Esteri? e l'ambasciata francese? E il cinema Mignon? Via del Babuino? Piazza Mastai? Amedeo rispondeva prima di Riccardo. Per quanto riguarda la conoscenza della storia di Roma, Amedeo non ha rivali, conosce l'origine dei nomi delle strade e i loro significati. Non ho mai visto in vita mia una persona come lui. Una volta, dopo l'ennesima sconfitta di fronte ad Amedeo, Riccardo gli ha detto ridendo: "Ammazza' Amede' come conosci Roma! Ma che t'ha allattato la lupa?"28

La struttura del romanzo prevede l'alternanza delle "deposizioni" degli altri undici personaggi, dette "verità", tra italiani e immigrati, agli "ululati" di Ahmed-Amedeo ed è proprio negli "ululati" che riemergono le problematiche identitarie del protagonista, che alla fine si domanda chi è in realtà:

Chi sono? Ahmed o Amedeo? Ah, Bágia! ${ }^{29}$

La nuova identità italiana non riesce completamente a sostituirsi ai ricordi dolorosi della memoria algerina e verso la fine del romanzo questi ricordi emergono prepotenti, assieme alla comparsa massiccia di vocaboli arabi non tradotti che evocano situazioni vissute da Ahmed-Amedeo in patria, rievocate anche in coincidenza con l'avvicinarsi del Ramadan (muezzin, buraq, qalb alluz, zlabia, maqrout, suhur, dhakar, zagharid). Bágia, si scopre posteriormente tramite il connazionale Abdallah Ben Kadour, significa "gioia" ed è anche il nome della città di Algeri.

D'altra parte, il collegamento tra identità e cibo, proposto nello stesso romanzo tramite il personaggio Parviz, sembra rispecchiare la stessa problematica. Parviz, iraniano di Shiraz, è l'esempio più chiaro della non integrazione. Come cuoco, è profondamente legato alla sua cultura gastronomica, che è poi il filo che lo unisce alla sua terra, quello che rimane della sua memoria, il ponte tra il passato e il presente ${ }^{30}$ :

Mi metto subito a preparare i vari piatti iraniani, come il gormeh sabzi e il kabab kubideh, i kashk badinjan e i kateh. Gli odori che riempiono la cucina mi fanno dimenticare la realtà e mi sembra di essere tornato nella mia cucina a Shiraz. Dopo un po' il profumo delle spezie si trasforma in incenso, ed è questo che mi fa ballare e cantare come un derviscio, ahi ahi ahi... Così in pochi minuti la cucina si trasforma in una trance sufi. ${ }^{31}$

Ahmed-Amedeo, nel suo primo "ululato", sostiene che Parviz si rifiuta di imparare la cucina italiana perché ha paura di dimenticare quella iraniana, cioè, di perdere la sua identità. Parviz è anche l'autore di una divertente condanna ai "pizzadipendenti", comelichiamalui. Coerentemente, Parviz 
detesta l'omologazione gastronomica e quindi i “pizzadipendenti”, tra i quali tuttavia anche l'amico Amedeo, cioè l'immigrato Ahmed, con un inconsapevole e rovesciato atteggiamento razzista:

Qualche giorno fa, non erano nemmeno le otto di mattina, seduto su un sedile della metropolitana, stropicciandomi gli occhi e lottando contro il sonno a causa di quel risveglio cosi mattiniero, ho visto una ragazza italiana che divorava una pizza grande come un ombrello. Mi è venuta la nausea e per poco non vomitavo! Grazie a Dio è scesa alla fermata successiva. Davvero una scena insopportabile! La legge dovrebbe punire chi si permette di turbare la tranquillità dei buoni cittadini che vanno al lavoro la mattina e tornano a casa la sera. Il danno provocato da chi mangia pizza in metropolitana supera di molto quello causato dalle sigarette. Spero che le autorità competenti non sottovalutino questa delicata questione e procedano immediatamente ad affiggere cartelli del tipo "Proibito mangiare la pizza" accanto a quelli che campeggiano all'ingresso delle gallerie della metro con la scritta "Vietato fumare!" Vorrei capire come fanno gli italiani a divorare una impressionante quantità di pasta mattina e sera.

Il mio odio per la pizza non ha paragoni, ma questo non significa che io odii tutte le persone che la mangiano. [...]

Amo molto Amedeo, nonostante sia un pizzadipendente. [...]

La mia preoccupazione è di evitare a tutti i costi le conseguenze dovute alla mia avversione alla pizza. Per esempio, qualche settimana fa mi hanno licenziato dal posto di lavapiatti in un ristorante vicino a piazza Navona quando hanno scoperto che odiavo la pizza. Figli di puttana. Dopo questo scandalo c'è ancora chi sostiene che la libertà di gusto, di espressione, di fede e la democrazia sono garantite in questo paese! Vorrei sapere: la legge punisce chi odia la pizza? Se la risposta è sì, siamo di fronte a un vero scandalo, se è no, allora io ho tutti i diritti di essere risarcito. ${ }^{32}$

Alla fine le questioni gastronomiche poste da Parviz si ricollegano alle questioni identitarie che emergono dal romanzo di Lakhous. Ahmed-Amedeo e Abdallah, entrambi algerini di Algeri, appartenenti allo stesso quartiere, vivono diversamente la questione dell'identità inserita in una nuova cultura. Mentre per Abdallah scatta una specie di resistenza contro qualsiasi forma di contaminazione o semplicemente di erosione della propria identità, con la continua riaffermazione dei valori della propria cultura di origine, per Ahmed-Amedeo invece prevale la celebrazione dell'incontro e della commistione, che per Abdallah diventa il simbolo negativo dello stravolgimento della tradizione. Nelle parole di Abdallah, la questione torna al primo contrassegno identitario, il nome: 
Perché si è fatto chiamare Amedeo? È questa la domanda che mi lascia molto perplesso. Il suo vero nome è Ahmed, un nome preziosissimo perché è uno di quelli del profeta Maometto e viene menzionato sia nel Corano che nel Vangelo. Francamente non apprezzo molto chi cambia il suo nome o rinnega le sue origini: ad esempio so che il mio nome è Abdallah, e so molto bene che è un nome difficile da pronunciare per gli italiani nonostante abbia giurato di non cambiarlo finché sono vivo. Non voglio disobbedire a mio padre, che mi ha dato questo nome, né a Dio, che ci ha vietato di disobbedire ai genitori. Cambiare nome è un peccato capitale come l'omicidio, l'adulterio, la falsa testimonianza, come derubare gli orfani. ${ }^{33}$

Il confronto antropologico sul nome si allarga naturalmente ad altri elementi, che qui possiamo solo accennare: sono gli stereotipi e i pregiudizi che gli italiani rivolgono agli immigrati ritrovati in altre opere della migrazione e di cui il romanzo è ampiamente dotato (rubano il lavoro ai loro figli; non si lavano; sono delinquenti e ignoranti), ma non manca il rovescio della medaglia, e cioè quelli degli immigrati verso gli italiani (sono razzisti; hanno sempre fretta; non conoscono l'islam, a cui applicano una serie di facili etichette). Nel primo caso, ad esempio, è notevole la riproposizione dell'equazione immigrato-delinquente associata ad Ahmed-Amedeo non più "italiano":

Quando hanno scoperto che è immigrato e non un italiano non hanno esitato ad accusarlo di omicidio. ${ }^{34}$

Talvolta il desiderio di un nome italiano viene ingenuamente percepito dallo straniero come un modo di inserirsi nel tessuto sociale, un modo di venire accettati come persone, come nel caso di Fitahianamalala Rakotobe Andriamaro, che nel racconto Chiamatemi Mina, premiato nella quinta edizione del concorso Eks\&Tra, racconta del suo desiderio di bambina di venire chiamata Mina a scuola, perché si vergognava del suo vero nome:

L'appello a scuola era un vero tormento. Sgranavo col respiro e col petto quell'elenco di rintocchi nitidi dal suono via via più forte fino all'apice, il mio nome, su cui la maestra avrebbe indugiato più che sugli altri, avrebbe faticato imbarazzata e al suo disagio si sarebbe aggiunto il mio. ${ }^{35}$

La problematica descritta dall'io narrante viene di solito risolta con un "Come ti chiamano di solito", seguito dalla risposta "Mina". L'io narrante, da bambina, non percepiva tale procedura, volta ad un atteggiamento conciliatorio e indulgente, come perdita di individualità e di complessità culturale: 
All'epoca dei fatti le mie priorità andavano ben oltre la salvaguardia del mio nome e, dopo tutto, che una tale mutilazione possa considerarsi una violenza alla mia identità culturale lo scopro solo oggi. La ragazzina di allora si preoccupava unicamente di rientrare nei canoni della norma [...].

Tra la pelle nera (la famiglia è di origine malgascia), la casa non arredata secondo idittami italianoemiliani, e quindi non in grado di essere esibita ai compagni di scuola “insindacabilmente emiliani", il padre che, nonostante la laurea in medicina, raccoglie frutta e si ostina a prendere la figlia a scuola "con la Prinz verde", la protagonista percepisce nel nome "uno scioglilingua" che rispecchiala difficile realtà della convivenza e dell'integrazione. Proprio per facilitare al figlio l'integrazione, il padre del protagonista del racconto "Solo allora, sono certo, potrò capire" di Tahar Lamri, pubblicato nella prima antologia del concorso Eks\&Tra, Le voci dell'arcobaleno (1995) e poi in I sessanta nomi dell'amore (Tracce diverse, 2007), lo chiama con un nome francese (il racconto è ambientato in Francia): JeanMarie. Il padre, di origini algerine, vieta al figlio qualsiasi contatto con la propria cultura:

Ecco, non conosco questo paese, perché semplicemente mio padre me lo ha sempre impedito. Diceva che questo paese non valeva la pena di essere visto... ${ }^{36}$

Non solo le visite al paese di origine del padre gli erano state impedite: il padre non gli aveva mai insegnato l'arabo e gli aveva vietato qualsiasi contatto con figli di immigrati. Questi accorgimenti miravano ad una perfetta integrazione del figlio nella società ospitante, la Francia:

Dice per il nome, vero? L'ha voluto mio padre, perché per lui faceva parte dell'integrazione, secondo lui... nostra, e, soprattutto, "mia" nella cultura francese. Lo sa una delle tante illusioni degli immigrati... ${ }^{37}$

L'integrazione rimane un'illusione: lo dimostra anche il fatto che il padre, dopo aver avuto un ictus, chiede di essere seppellito nel suo villaggio natale - "Non voglio essere sepolto nel paese degli altri", afferma in quel momento doloroso. Il paese dell'immigrazione, sia pure vissuto come patria d'elezione - "I diecimila ettari di Parigi sono il mio regno"38 -, resta comunque "il paese degli altri”. Quando il protagonista si reca quindi per la prima volta in Algeria, dopo la morte del padre, seppur abituato alle comodità parigine, ne rimane affascinato:

Fatto sta che mi sono innamorato di quel villaggio, con le sue case di terra, la sua nudità, le sue piste sonnolente sotto il sole, i suoi sudici bambini con le candele al naso, i suoi ciuffi d'erba secca in mezzo ai sassi, i passi furtivi delle donne. Poi, oggi, la vostra ospitalità. Tante cose. ${ }^{39}$

36. Tahar Lamri, "Solo allora, sono certo, potrò capire", in Le voci dell'arcobaleno, cit., p. 51.

37. Ibidem, p. 37.

38. Ibidem, p. 52.

39. Ibidem, p. 53. 
Durante la cena che condivide con una famiglia algerina, dopo che il suo volo per Parigi viene rimandato al giorno seguente, il protagonista si ricongiunge con quella parte del sé che il padre gli aveva impedito di far emergere, imponendogli un'identità francese; alla fine, il peso delle radici verrà prepotentemente a galla quando lui deciderà di stabilirsi definitivamente in quel paese:

Jean-Marie si meravigliava dell'abbondanza dei piatti, sapientemente disposti. Tutto era messo lì: la zuppa, la carne, l'insalata, il dolce, la frutta; altrettanto erano abbondanti i colori e i profumi. L'aria della stanza era satura degli odori di cardamomo, cumino, carvi, pepe e di altre spezie. Jean-Marie bevve la profumatissima chorba, assaporò lentamente la tenerissima carne di pollo, riprese dell'agnello dolce spruzzato di acqua di fiori d'arancio. Mangiava in raccoglimento aiutato dal silenzio degli altri.

Gli sembrava di essere in viaggio, nel viaggio del ritorno a sé. ${ }^{40}$

Nel primo impatto con la società di accoglienza, la salvaguardia del proprio nome appare come una misura necessaria contro l'appropriazione o la sottrazione di un "pezzo" identitario, come nelle parole di Karim di Fiamme in paradiso (2000). Il nome è quindi nella letteratura migrante una, e forse la più evidente, tra le misure dell'integrazione. Le opposte propensioni ad una non facile integrazione e alla difesa della propria, spesso problematica, identità culturale rappresentano il primo raffronto dell'esperienza culturale del migrante, anche prima del confronto con l'autoctono come altro da sé. Tant’è vero che a uno dei più ironici rappresentanti della letteratura migrante, Lakhous, riesce più facile mettere in ridicolo i pregiudizi degli italiani contro gli immigrati che risolvere l'ambivalente sentimento di appartenenza del protagonista.

Nel romanzo Scontro di civiltà per un ascensore a Piazza Vittorio, la portinaia napoletana Benedetta Esposito riproduce alcuni dei luoghi comuni che normalmente vengono attribuiti alla figura dell'immigrato: sono delinquenti, rubano lavoro agli italiani, portano malattie; ma non solo, la sua concezione generale dello straniero comprende anche la loro regione d'origine, ovunque essa sia, sempre caratterizzata dagli stessischemi: vivono all'aperto o in tende, mangiano conle mani, trattano le donne come schiave e si spostano con asini o cammelli ${ }^{41}$. Sono tratti semplificatori che alludono alla stessa forma di appiattimento che porta, in altre varianti, alla generalizzazione della figura dell'immigrato come "marocchino" o "vu' cumprà". Da una parte sono termini che costruiscono il meccanismo della differenza, e dall'altra diventano strumenti dipratiche escludenti ediscriminatorie. Lo stesso personaggio poc'anzi citato sentenzia: "Vivere con loro è impossibile"42

Non è difficile riconoscere in queste strategie i modi con cui Zygmunt Bauman, prendendo in prestito concetti di Lévi-Strauss, spiega i meccanismi della "guerra" moderna contro lo straniero mediante due strategie diverse: antropofagica e antropoemica. Nella prima, lo straniero

40. Ibidem, p. 49.

41. A. Lakhous, op. cit., pp. 47-51.

42. Ibidem, p. 50. 
viene annichilato, divorato e poi trasformato in tessuto indistinguibile dal proprio: si tratta dell'assimilazione, e cioè, di rendere simile il dissimile; la seconda invece rigetta lo straniero, lo allontana, lo segrega e lo espelle: è la strategia dell'esclusione; lo straniero viene rinchiuso all'interno dei muri visibili dei ghetti o dietro quelli invisibili dai divieti del "commercium, commensalium e conubium"; quando necessario viene eliminato fisicamente ${ }^{43}$.

Il migrante può decidere di diventare invisibile (come Ahmed/Amedeo o Jean-Marie, suo malgrado), assimilandosi alla società ospitante e assumendone i connotati (lingua, cibo, nome, conoscenza del territorio), quando gli è permesso di farlo, o di opporsi all'assimilazione (Abdallah, Mohamed), mettendo in discussione le regole di questa società e coltivando la differenza.

D'altronde, le forme di resistenza possono essere anch'esse diverse. Per Abdallah essa si concretizza nella non accettazione della contaminazione e nella riaffermazione dei propri valori e precetti; per Parviz essa significa un attaccamento esageratamente nostalgico alla propria cultura, con il rifiuto categorico della cultura dell'altro, diventata oggetto di sentimenti di odio (simboleggiato dall'odio per la pizza); per Mohamed si tratta di eleggere un compromesso parziale per preservare un tratto essenziale dell'identità (araba/islamica). Entrambi gli atteggiamenti - resistenza/assimilazione - s'identificano con la dialettica delle identità diStuart Hall, sintetizzate nella questione traduzione/ tradizione, in cui la prima punta all'attraversamento di frontiere ela formazione di nuove identità in un processo di continua negoziazione; la seconda invece si riferisce alla chiusura alla contaminazione e al desiderio di restaurare la purezza originale ${ }^{44}$; entrambe, se esasperate, possono condurre a espressioni degenerate: la prima può portare all'annullamento dei tratti identitari essenziali e all'omogeneizzazione culturale, mentre invece la seconda può avere esiti drammatici, se tradotta in meccanismi fondamentalisti, come avverte Amin Maalouf:

Ciascuno di noi dovrebbe essere incoraggiato ad assumere la propria diversità, a concepire la propria identità come la somma delle sue diverse appartenenze, invece di confonderla con una sola, eretta ad appartenenza suprema e a strumento di esclusione, talvolta a strumento di guerra. ${ }^{45}$

43. Zygmunt Bauman, Il disagio della postmodernità, trad. Vera Verdiani, Milano, Bruno Mondadori, 2002, p. 22.

44. Cfr. Stuart Hall, "The Question of Cultural Identity", in S. Hall, D. Held, T. McGrew (editors), Modernity and its Futures, Cambridge, Polity Press, 1992, pp. 274-325.

45. Amin Maalouf, L'identità, trad. Fabrizio Ascari, Milano, Bompiani, 1999, p. 6. 